\title{
A Matrix Lie Superalgebra and Its Applications
}

\author{
Jingwei Han, ${ }^{1}$ Jing $\mathrm{Yu}^{2}{ }^{2}$ and Jingsong $\mathrm{He}^{3}$ \\ ${ }^{1}$ School of Information Engineering, Hangzhou Dianzi University, Hangzhou, Zhejiang 310018, China \\ ${ }^{2}$ School of Science, Hangzhou Dianzi University, Hangzhou, Zhejiang 310018, China \\ ${ }^{3}$ School of Science, Ningbo University, Ningbo, Zhejiang 315211, China
}

Correspondence should be addressed to Jingsong He; hejingsong@nbu.edu.cn

Received 24 August 2013; Accepted 30 November 2013

Academic Editor: Xing Biao Hu

Copyright (C) 2013 Jingwei Han et al. This is an open access article distributed under the Creative Commons Attribution License, which permits unrestricted use, distribution, and reproduction in any medium, provided the original work is properly cited.

A matrix Lie superalgebra is established. As its applications, multicomponent super Ablowitz-Kaup-Newell-Segur (AKNS) equations and multicomponent super Dirac equations are constructed. By making use of supertrace identity, their superHamiltonian structures are presented, respectively.

\section{Introduction}

It is well-known that by making use of the trace identity, many integrable equations can be written as the Hamiltonian forms, which was first proposed by Tu in [1]. The key ideas include the following three aspects. Firstly, from the finitedimensional Lie algebra

$$
G=\left\{E_{1}, \ldots, E_{d}\right\},
$$

Tu constructs a loop Lie algebra

$$
\widetilde{G}=\left\{E_{1}(n), \ldots, E_{d}(n)\right\},
$$

where $E_{i}(n)=E_{i} \otimes \lambda^{n}=E_{i} \lambda^{n}$. Secondly, an iso-spectral problem $\left(\lambda_{t_{n}}=0\right)$

$$
\psi_{x}=U(u, \lambda) \psi
$$

is considered, where $\lambda$ is a spectral parameter and $u$ is a potential. By solving the adjoint representation equation

$$
V_{x}=[U, V]
$$

and zero curvature equations

$$
U_{t_{n}}-V_{x}^{(n)}+\left[U, V^{(n)}\right]=0, \quad V^{(n)}=\left(\lambda^{n} V\right)_{+},
$$

where plus denotes the choice of the nonnegative power of $\lambda$, Tu obtains integrable equations. Lastly, by using the trace identity

$$
\frac{\delta}{\delta u}\left\langle V, \frac{\partial U}{\partial \lambda}\right\rangle=\left(\lambda^{-\gamma} \frac{\partial}{\partial \lambda} \lambda^{\gamma}\right)\left\langle V, \frac{\partial U}{\partial u}\right\rangle,
$$

where $\gamma$ is a undetermined constant, the obtained integrable equations can be written as the Hamiltonian form. Some wellknown integrable Hamiltonian equations have been obtained by this method, such as AKNS equations, Kaup-Newll (KN) equations, and Wadati-Konno-Ichikawa (WKI) equations.

This method for constructing Hamiltonian structures of integrable equations was extended to superintegrable equations by $\mathrm{Hu}$ in [2], where the supertrace identity

$$
\frac{\delta}{\delta u} \int \operatorname{str}\left(V \frac{\partial U}{\partial \lambda}\right) d x=\left(\lambda^{-\gamma} \frac{\partial}{\partial \lambda} \lambda^{\gamma}\right) \operatorname{str}\left(\frac{\partial U}{\partial u} V\right)
$$

was first proposed by $\mathrm{Hu}$ in [3] and proved by $\mathrm{Ma}$ et al. in [4]. Similarly, many integrable super-Hamiltonian equations have also been constructed, such as super AKNS equations, super Dirac equations [4], super coupled Korteweg-de Vries $(\mathrm{cKdV})$ equations $[5,6]$, and super $\mathrm{KN}$ equations $[7,8]$.

It is a valuable generalization from one component to multicomponent in soliton equations because multicomponent soliton equations possess more complex structure and become more extensive than one-component ones. In physics, multicomponent integrable system is widely applied. 
For example, mixed N-coupled nonlinear Schrödinger equations (NLS), two-component Bose-Einstein condensates (BECs), and coupled Schrödinger equation are, respectively, discussed in [9-11], and they obtain many new results for these multicomponent equations. In mathematics, the inverse scattering method provides us a powerful tool to find multicomponent extensions of one-component soliton equations, like multicomponent AKNS equations [12, 13], multicomponent NLS equation [14], and so on [15-18]. Moreover, from the point of mathematics, we find that multicomponent generalizations mainly include the following aspects:

(1) symmetric space $[14,19,20]$,

(2) matrix algebra [13, 21-23],

(3) soliton hierarchy associated with a matrix pseudodifferential operator $[24,25]$.

Based on results from the above analysis, some questions are listed as follows.

(1) Do multicomponent superintegrable equations exist?

(2) If they exist, how can the multicomponent superintegrable equations be constructed?

(3) By making use of the supertrace identity (7), can multicomponent superintegrable equations be written as the super-Hamiltonian form?

The purpose of this paper is to answer these questions. The paper is organized as follows. In the next section, we construct a matrix Lie superalgebra $A(2 m-1, m-1)$ [26]. As its applications, multicomponent super AKNS equations and multicomponent super Dirac equations are, respectively, constructed in Sections 3 and 4. Their super bi-Hamiltonian forms are also, respectively, constructed in these sections. Some conclusions and discussions are listed in the last section.

\section{A Matrix Lie Superalgebra}

Let us start with the following linear space $G=\left\{e_{1}, e_{2}, e_{3}\right.$, $\left.e_{4}, e_{5}\right\}$ :

$$
\begin{aligned}
& e_{1}=\left(\begin{array}{ccc}
E & O & O \\
O & -E & O \\
O & O & O
\end{array}\right), \quad e_{2}=\left(\begin{array}{ccc}
O & E & O \\
O & O & O \\
O & O & O
\end{array}\right), \\
& e_{3}=\left(\begin{array}{ccc}
O & O & O \\
E & O & O \\
O & O & O
\end{array}\right), \quad e_{4}=\left(\begin{array}{ccc}
O & O & E \\
O & O & O \\
O & -E & O
\end{array}\right) \text {, } \\
& e_{5}=\left(\begin{array}{ccc}
O & O & O \\
O & O & E \\
E & O & O
\end{array}\right) \\
& {\left[e_{1}, e_{2}\right\}=2 e_{2}, \quad\left[e_{1}, e_{3}\right\}=-2 e_{3} \text {, }} \\
& {\left[e_{2}, e_{3}\right\}=e_{1}, \quad\left[e_{1}, e_{4}\right\}=e_{4} \text {, }} \\
& {\left[e_{1}, e_{5}\right\}=-e_{5}, \quad\left[e_{2}, e_{4}\right\}=\left[e_{3}, e_{5}\right\}=0 \text {, }} \\
& {\left[e_{2}, e_{5}\right\}=e_{4}, \quad\left[e_{3}, e_{4}\right\}=e_{5},}
\end{aligned}
$$

$$
\begin{gathered}
{\left[e_{4}, e_{4}\right\}=-2 e_{2}, \quad\left[e_{4}, e_{5}\right\}=e_{1},} \\
{\left[e_{5}, e_{5}\right\}=2 e_{3},}
\end{gathered}
$$

where $E$ is a $m \times m$ unit matrix, $O$ is a $m \times m$ zero matrix, $G_{0}=\left\{e_{1}, e_{2}, e_{3}\right\}$ is even, $G_{1}=\left\{e_{4}, e_{5}\right\}$ is odd, $[a, b\}=a b-$ $(-1)^{p(a) p(b)} b a$ is the super Lie bracket, and $p(f)$ denotes the parity of the arbitrary element $f$.

It is easy to prove that the linear space $G$ is a matrix Lie superalgebra $A(2 m-1, m-1)$. The corresponding loop superalgebra $\widetilde{G}$ is presented as

$$
\begin{gathered}
e_{i}(n)=e_{i} \lambda^{n}, \quad\left[e_{1}(m), e_{2}(n)\right\}=2 e_{2}(m+n), \\
{\left[e_{1}(m), e_{3}(n)\right\}=-2 e_{3}(m+n),} \\
{\left[e_{2}(m), e_{3}(n)\right\}=e_{1}(m+n),} \\
{\left[e_{1}(m), e_{4}(n)\right\}=e_{4}(m+n),} \\
{\left[e_{1}(m), e_{5}(n)\right\}=-e_{5}(m+n),} \\
{\left[e_{2}(m), e_{4}(n)\right\}=\left[e_{3}(m), e_{5}(n)\right\}=0,} \\
{\left[e_{2}(m), e_{5}(n)\right\}=e_{4}(m+n),} \\
{\left[e_{3}(m), e_{4}(n)\right\}=e_{5}(m+n),} \\
{\left[e_{4}(m), e_{4}(n)\right\}=-2 e_{2}(m+n),} \\
{\left[e_{4}(m), e_{5}(n)\right\}=e_{1}(m+n),} \\
{\left[e_{5}(m), e_{5}(n)\right\}=2 e_{3}(m+n),} \\
\operatorname{deg} e_{i}(n)=n, \quad(i=1,2,3,4,5) .
\end{gathered}
$$

\section{Multicomponent Super AKNS Hierarchy and Its Super-Hamiltonian Structure}

In this section, we will derive multicomponent super AKNS hierarchy by the above matrix Lie superalgebra, and further, its super-Hamiltonian structure will be constructed.

Let us consider the following spectral problem:

$$
\begin{aligned}
\phi_{x}= & U(u, \lambda) \phi, \\
U= & -e_{1}(1)+v e_{2}(0)+w e_{3}(0) \\
& +\alpha e_{4}(0)+\beta e_{5}(0),
\end{aligned}
$$

which can be written in the following matrix form:

$$
\phi_{x}=U(u, \lambda) \phi=\left(\begin{array}{ccc}
-\lambda E & v & \alpha \\
w & \lambda E & \beta \\
\beta & -\alpha & 0
\end{array}\right) \phi,
$$

where $v=\operatorname{diag}\left(v_{1}, \ldots, v_{m}\right), w=\operatorname{diag}\left(w_{1}, \ldots, w_{m}\right), \alpha=$ $\operatorname{diag}\left(\alpha_{1}, \ldots, \alpha_{m}\right), \beta=\operatorname{diag}\left(\beta_{1}, \ldots, \beta_{m}\right), u=(v, w, \alpha, \beta)^{T}$, $p(\lambda)=p\left(v_{j}\right)=p\left(w_{j}\right)=0$, and $p\left(\alpha_{j}\right)=p\left(\beta_{j}\right)=1$ $(1 \leq j \leq m)$. Note that $U(u, \lambda) \in A(2 m-1, m-1)$. Taking

$$
V=\left(\begin{array}{ccc}
a & b & \rho \\
c & -a & \delta \\
\delta & -\rho & 0
\end{array}\right),
$$


where $a=\operatorname{diag}\left(a_{1}, \ldots, a_{m}\right), b=\operatorname{diag}\left(b_{1}, \ldots, b_{m}\right), c=$ $\operatorname{diag}\left(c_{1}, \ldots, c_{m}\right), \rho=\operatorname{diag}\left(\rho_{1}, \ldots, \rho_{m}\right)$, and $\delta=$ $\operatorname{diag}\left(\delta_{1}, \ldots, \delta_{m}\right)$, the adjoint representation equation (4) gives

$$
\begin{aligned}
& a_{k, x}=v_{k} c_{k}-w_{k} b_{k}+\alpha_{k} \delta_{k}+\beta_{k} \rho_{k}, \\
& b_{k, x}=-2 \lambda b_{k}-2 v_{k} a_{k}-2 \alpha_{k} \rho_{k}, \\
& c_{k, x}=2 \lambda c_{k}+2 w_{k} a_{k}+2 \beta_{k} \delta_{k}, \\
& \rho_{k, x}=-\lambda \rho_{k}+v_{k} \delta_{k}-\alpha_{k} a_{k}-\beta_{k} b_{k}, \\
& \delta_{k, x}=\lambda \delta_{k}+w_{k} \rho_{k}-\alpha_{k} c_{k}+\beta_{k} a_{k},
\end{aligned}
$$

where $1 \leq k \leq m$. Let $a_{k}=\sum_{j \geq 0} a_{k}^{(j)} \lambda^{-j}, b_{k}=\sum_{j \geq 0} b_{k}^{(j)} \lambda^{-j}$, $c_{k}=\sum_{j \geq 0} c_{k}^{(j)} \lambda^{-j}, \rho_{k}=\sum_{j \geq 0} \rho_{k}^{(j)} \lambda^{-j}$, and $\delta_{k}=\sum_{j \geq 0} \delta_{k}^{(j)} \lambda^{-j}$, and then (13) becomes

$$
\begin{gathered}
b_{k}^{(0)}=c_{k}^{(0)}=\rho_{k}^{(0)}=\delta_{k}^{(0)}=0, \\
a_{k, x}^{(j)}=v_{k} c_{k}^{(j)}-w_{k} b_{k}^{(j)}+\alpha_{k} \delta_{k}^{(j)}+\beta_{k} \rho_{k}^{(j)}, \quad j \geq 0, \\
b_{k, x}^{(j)}=-2 b_{k}^{(j+1)}-2 v_{k} a_{k}^{(j)}-2 \alpha_{k} \rho_{k}^{(j)}, \quad j \geq 0, \\
c_{k, x}^{(j)}=2 c_{k}^{(j+1)}+2 w_{k} a_{k}^{(j)}+2 \beta_{k} \delta_{k}^{(j)}, \quad j \geq 0, \\
\rho_{k, x}^{(j)}=-\rho_{k}^{(j+1)}+v_{k} \delta_{k}^{(j)}-\alpha_{k} a_{k}^{(j)}-\beta_{k} b_{k}^{(j)}, \quad j \geq 0, \\
\delta_{k, x}^{(j)}=\delta_{k}^{(j+1)}+w_{k} \rho_{k}^{(j)}-\alpha_{k} c_{k}^{(j)}+\beta_{k} a_{k}^{(j)}, \quad j \geq 0,
\end{gathered}
$$

where $1 \leq k \leq m$. Moreover, we find that (14) can be written as the following recursive form:

$$
\left(\begin{array}{ccc}
c_{1}^{(j+1)} & & \\
& \ddots & \\
b_{1}^{(j+1)} & & c_{m}^{(j+1)} \\
& \ddots & \\
2 \delta_{1}^{(j+1)} & & b_{m}^{(j+1)} \\
& \ddots & \\
-2 \rho_{1}^{(j+1)} & & 2 \delta_{m}^{(j+1)} \\
& \ddots & \\
& -2 \rho_{m}^{(j+1)}
\end{array}\right)
$$

where the recursive operator is given by

$$
\mathscr{L}=\left(\begin{array}{llll}
\mathscr{L}_{11} & \mathscr{L}_{12} & \mathscr{L}_{13} & \mathscr{L}_{14} \\
\mathscr{L}_{21} & \mathscr{L}_{22} & \mathscr{L}_{23} & \mathscr{L}_{24} \\
\mathscr{L}_{31} & \mathscr{L}_{32} & \mathscr{L}_{33} & \mathscr{L}_{34} \\
\mathscr{L}_{41} & \mathscr{L}_{42} & \mathscr{L}_{43} & \mathscr{L}_{44}
\end{array}\right),
$$

where

$$
\begin{gathered}
\mathscr{L}_{11}=\operatorname{diag}\left(\frac{1}{2} \partial-w_{1} \partial^{-1} v_{1}, \ldots, \frac{1}{2} \partial-w_{m} \partial^{-1} v_{m}\right), \\
\mathscr{L}_{12}=\operatorname{diag}\left(w_{1} \partial^{-1} w_{1}, \ldots, w_{m} \partial^{-1} w_{m}\right), \\
\mathscr{L}_{13}=\operatorname{diag}\left(-\frac{1}{2} \beta_{1}-\frac{1}{2} w_{1} \partial^{-1} \alpha_{1}, \ldots,\right. \\
\left.\quad-\frac{1}{2} \beta_{m}-\frac{1}{2} w_{m} \partial^{-1} \alpha_{m}\right), \\
\mathscr{L}_{14}=\operatorname{diag}\left(\frac{1}{2} w_{1} \partial^{-1} \beta_{1}, \ldots, \frac{1}{2} w_{m} \partial^{-1} \beta_{m}\right), \\
\mathscr{L}_{21}=\operatorname{diag}\left(-v_{1} \partial^{-1} v_{1}, \ldots,-v_{m} \partial^{-1} v_{m}\right), \\
\mathscr{L}_{22}=\operatorname{diag}\left(-\frac{1}{2} \partial+v_{1} \partial^{-1} w_{1}, \ldots,-\frac{1}{2} \partial+v_{m} \partial^{-1} w_{m}\right), \\
\mathscr{L}_{23}=\operatorname{diag}\left(-\frac{1}{2} v_{1} \partial^{-1} \alpha_{1}, \ldots,-\frac{1}{2} v_{m} \partial^{-1} \alpha_{m}\right), \\
\mathscr{L}_{24}=\operatorname{diag}\left(\frac{1}{2} \alpha_{1}+\frac{1}{2} v_{1} \partial^{-1} \beta_{1}, \ldots, \frac{1}{2} \alpha_{m}+\frac{1}{2} v_{m} \partial^{-1} \beta_{m}\right), \\
\mathscr{L}_{31}=\operatorname{diag}\left(2 \alpha_{1}-2 \beta_{1} \partial^{-1} v_{1}, \ldots, 2 \alpha_{m}-2 \beta_{m} \partial^{-1} v_{m}\right), \\
\mathscr{L}_{32}=\operatorname{diag}\left(2 \beta_{1} \partial^{-1} w_{1}, \ldots, 2 \beta_{m} \partial^{-1} w_{m}\right), \\
\mathscr{L}_{33}=\operatorname{diag}\left(\partial-\beta_{1} \partial^{-1} \alpha_{1}, \ldots, \partial-\beta_{m} \partial^{-1} \alpha_{m}\right), \\
\mathscr{L}_{34}=\operatorname{diag}\left(w_{1}+\beta_{1} \partial^{-1} \beta_{1}, \ldots, w_{m}+\beta_{m} \partial^{-1} \beta_{m}\right), \\
\mathscr{L}_{41}=\operatorname{diag}\left(2 \alpha_{1} \partial^{-1} v_{1}, \ldots, 2 \alpha_{m} \partial^{-1} v_{m}\right), \\
\mathscr{L}_{42}=\operatorname{diag}\left(2 \beta_{1}-2 \alpha_{1} \partial^{-1} w_{1}, \ldots, 2 \beta_{m}-2 \alpha_{m} \partial^{-1} w_{m}\right), \\
\mathscr{L}_{43}=\operatorname{diag}\left(-v_{1}+\alpha_{1} \partial^{-1} \alpha_{1}, \ldots,-v_{m}+\alpha_{m} \partial^{-1} \alpha_{m}\right), \\
\mathscr{L}_{44}=\operatorname{diag}\left(-\partial-\alpha_{1} \partial^{-1} \beta_{1}, \ldots,-\partial-\alpha_{m} \partial^{-1} \beta_{m}\right) .
\end{gathered}
$$

After a direct calculation, we obtain that $a_{k, x}^{(0)}=0$. Choosing $a_{k}^{(0)}=-1(1 \leq k \leq m)$ and constant of integration to be zero, the first few terms are listed as follows:

$$
\begin{gathered}
a_{k}^{(1)}=0, \quad b_{k}^{(1)}=v_{k}, \quad c_{k}^{(1)}=w_{k}, \\
\rho_{k}^{(1)}=\alpha_{k}, \quad \delta_{k}^{(1)}=\beta_{k}, \\
a_{k}^{(2)}=\frac{1}{2} v_{k} w_{k}+\alpha_{k} \beta_{k}, \quad b_{k}^{(2)}=-\frac{1}{2} v_{k, x}, \\
c_{k}^{(2)}=\frac{1}{2} w_{k, x}, \quad \rho_{k}^{(2)}=-\alpha_{k, x}, \quad \delta_{k}^{(2)}=\beta_{k, x},
\end{gathered}
$$

where $1 \leq k \leq m$. 
Then, let us consider the spectral problem (11) with the following auxiliary spectral problem:

$$
\phi_{t_{n}}=V^{(n)} \phi, \quad V^{(n)}=\left(\lambda^{n} V\right)_{+} .
$$

The compatibility conditions of (11) and (19), that is, the zero curvature equations (5) give an infinite hierarchy of nonlinear partial differential equations:

$$
\begin{array}{ll}
v_{k, t_{n}}=-2 b_{k}^{(n+1)}, & 1 \leq k \leq m, \\
w_{k, t_{n}}=2 c_{k}^{(n+1)}, & 1 \leq k \leq m, \\
\alpha_{k, t_{n}}=-\rho_{k}^{(n+1)}, & 1 \leq k \leq m, \\
\beta_{k, t_{n}}=\delta_{k}^{(n+1)}, & 1 \leq k \leq m,
\end{array}
$$

or

$$
\begin{array}{cc}
v_{t_{n}}=-2 b^{(n+1)}, & w_{t_{n}}=2 c^{(n+1)}, \\
\alpha_{t_{n}}=-\rho^{(n+1)}, & \beta_{t_{n}}=\delta^{(n+1)},
\end{array}
$$

where $a^{(j)}=\operatorname{diag}\left(a_{1}^{(j)}, \ldots, a_{m}^{(j)}\right), b^{(j)}=\operatorname{diag}\left(b_{1}^{(j)}, \ldots, b_{m}^{(j)}\right)$, $c^{(j)}=\operatorname{diag}\left(c_{1}^{(j)}, \ldots, c_{m}^{(j)}\right), \rho^{(j)}=\operatorname{diag}\left(\rho_{1}^{(j)}, \ldots, \rho_{m}^{(j)}\right), \delta^{(j)}=$ $\operatorname{diag}\left(\delta_{1}^{(j)}, \ldots, \delta_{m}^{(j)}\right)$, with $j \geq 0$. When $m=1$, (21) is equivalent to the super AKNS soliton hierarchy [27-30], and thus (21) is called multicomponent super AKNS hierarchy.

In what follows, a super-Hamiltonian structure of (21) is derived by means of the supertrace identity (7). To this end, the following quantities are needed:

$$
\begin{gathered}
\operatorname{str}\left(V \frac{\partial U}{\partial \lambda}\right)=-2 \text { tra, } \quad \operatorname{str}\left(\frac{\partial U}{\partial v} V\right)=c, \\
\operatorname{str}\left(\frac{\partial U}{\partial w} V\right)=b, \quad \operatorname{str}\left(\frac{\partial U}{\partial \alpha} V\right)=2 \delta, \\
\operatorname{str}\left(\frac{\partial U}{\partial \beta} V\right)=-2 \rho .
\end{gathered}
$$

Thus, the supertrace identity (7) gives the following equality:

$$
\left(\begin{array}{ccc}
\frac{\delta}{\delta v_{1}} & & \\
& \ddots & \frac{\delta}{\delta v_{m}} \\
\frac{\delta}{\delta w_{1}} & \ddots & \\
& & \frac{\delta}{\delta w_{m}} \\
\frac{\delta}{\delta \alpha_{1}} & \ddots & \\
\frac{\delta}{\delta \beta_{1}} & & \\
& & \frac{\delta}{\delta \alpha_{m}} \\
& & \\
& & \\
\delta \beta_{m}
\end{array} \sum_{k=1}^{m} a_{k}^{(j)} \lambda^{-j} d x\right.
$$

Equating the coefficients of $\lambda^{-j-2}$ on two sides of the above equality, we have

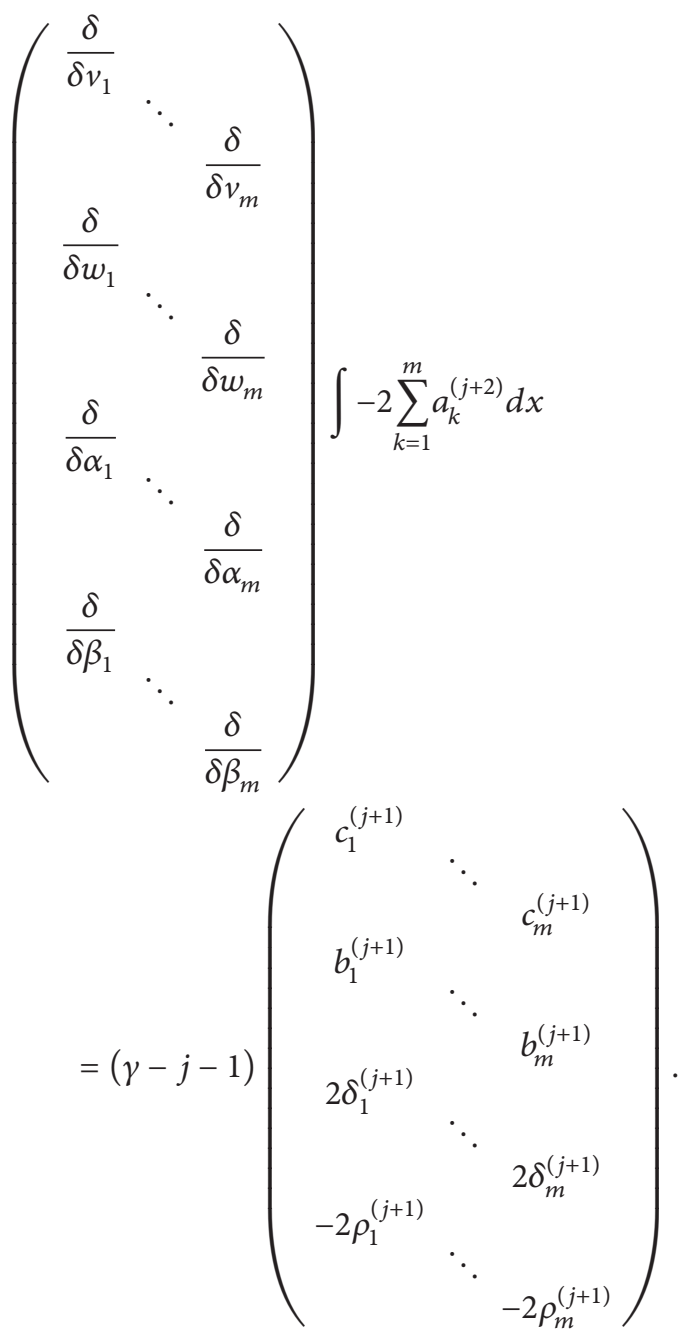


By taking $j=0$, we obtain that the constant $\gamma=0$. Thus, we have

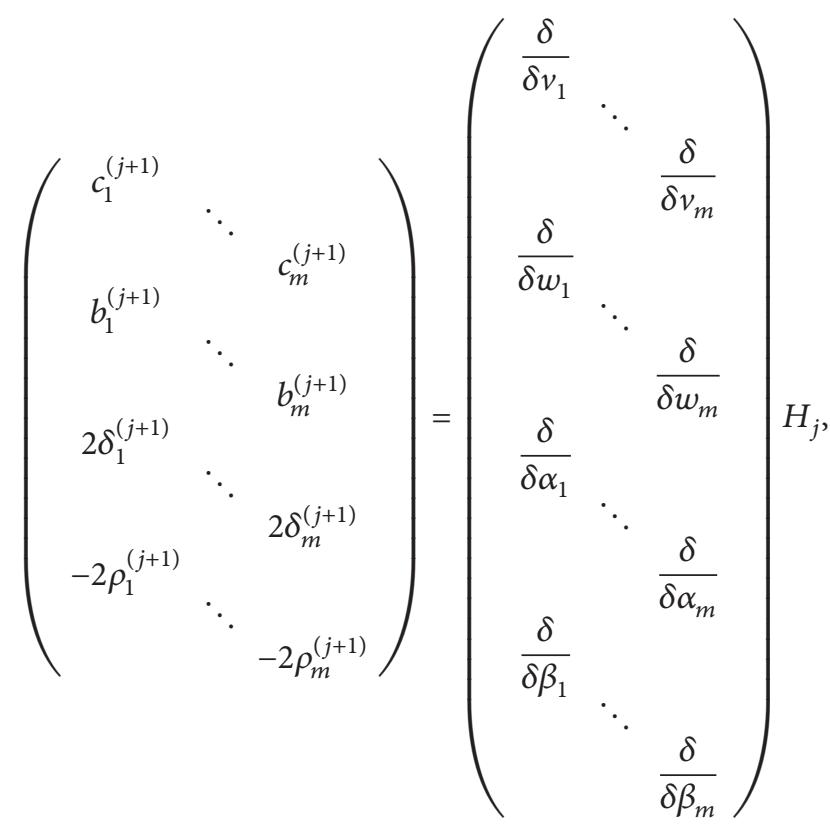

where $H_{j}=\int(2 /(j+1)) \sum_{k=1}^{m} a_{k}^{(j+2)} d x$.

Therefore, (21) can be written in the following super biHamiltonian form:

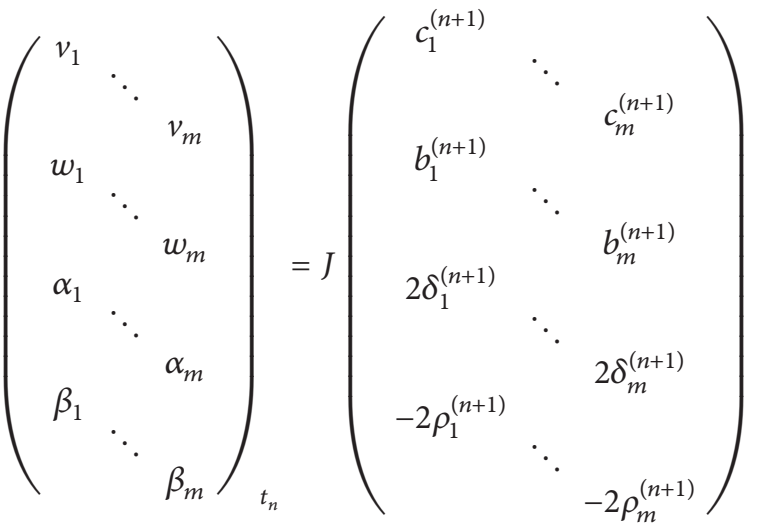

$$
\begin{aligned}
& =J \frac{\delta}{\delta u} H_{n}=J \mathscr{L} \frac{\delta}{\delta u} H_{n-1},
\end{aligned}
$$

where $J=\left(\begin{array}{cccc}0 & -2 E & 0 & 0 \\ 2 E & 0 & 0 & 0 \\ 0 & 0 & 0 & (1 / 2) E \\ 0 & 0 & (1 / 2) E & 0\end{array}\right)$ is the supersymplectic operator.

Example 1. Let $m=2, n=2$ in (21), and we have

$$
\begin{aligned}
& v_{1, t_{2}}=-\frac{1}{2} v_{1, x x}+v_{1}^{2} w_{1}+2 v_{1} \alpha_{1} \beta_{1}-2 \alpha_{1} \alpha_{1, x} \\
& v_{2, t_{2}}=-\frac{1}{2} v_{2, x x}+v_{2}^{2} w_{2}+2 v_{2} \alpha_{2} \beta_{2}-2 \alpha_{2} \alpha_{2, x}
\end{aligned}
$$

$$
\begin{aligned}
& w_{1, t_{2}}=\frac{1}{2} w_{1, x x}-v_{1} w_{1}^{2}-2 w_{1} \alpha_{1} \beta_{1}-2 \beta_{1} \beta_{1, x} \\
& w_{2, t_{2}}=\frac{1}{2} w_{2, x x}-v_{2} w_{2}^{2}-2 w_{2} \alpha_{2} \beta_{2}-2 \beta_{2} \beta_{2, x} \\
& \alpha_{1, t_{2}}=-\alpha_{1, x x}-v_{1} \beta_{1, x}+\frac{1}{2} v_{1} w_{1} \alpha_{1}-\frac{1}{2} v_{1, x} \beta_{1}, \\
& \alpha_{2, t_{2}}=-\alpha_{2, x x}-v_{2} \beta_{2, x}+\frac{1}{2} v_{2} w_{2} \alpha_{2}-\frac{1}{2} v_{2, x} \beta_{2}, \\
& \beta_{1, t_{2}}=\beta_{1, x x}+w_{1} \alpha_{1, x}+\frac{1}{2} w_{1, x} \alpha_{1}-\frac{1}{2} v_{1} w_{1} \beta_{1}, \\
& \beta_{2, t_{2}}=\beta_{2, x x}+w_{2} \alpha_{2, x}+\frac{1}{2} w_{2, x} \alpha_{2}-\frac{1}{2} v_{2} w_{2} \beta_{2},
\end{aligned}
$$

which is the first nonlinear two-component super AKNS equations.

\section{MultiComponent Super Dirac Hierarchy and Its Super-Hamiltonian Structure}

As another application of the matrix Lie superalgebra, multicomponent super Dirac hierarchy will be constructed, and further, its super-Hamiltonian structure will also be obtained.

Let us consider the following spectral problem:

$$
\begin{gathered}
\phi_{x}=U(\tilde{u}, \lambda) \phi, \\
U=e_{2}(1)-e_{3}(1)+r e_{1}(0)+s e_{2}(0) \\
+s e_{3}(0)+\alpha e_{4}(0)+\beta e_{5}(0),
\end{gathered}
$$

which can be written in the following matrix form:

$$
\phi_{x}=U(\widetilde{u}, \lambda) \phi=\left(\begin{array}{ccc}
r & \lambda E+s & \alpha \\
-\lambda E+s & -r & \beta \\
\beta & -\alpha & 0
\end{array}\right) \phi
$$

where $r=\operatorname{diag}\left(r_{1}, \ldots, r_{m}\right), s=\operatorname{diag}\left(s_{1}, \ldots, s_{m}\right), \alpha=$ $\operatorname{diag}\left(\alpha_{1}, \ldots, \alpha_{m}\right), \beta=\operatorname{diag}\left(\beta_{1}, \ldots, \beta_{m}\right), \tilde{u}=(r, s, \alpha, \beta)^{T}$, $p(\lambda)=p\left(r_{i}\right)=p\left(s_{i}\right)=0$, and $p\left(\alpha_{i}\right)=p\left(\beta_{i}\right)=1(1 \leq i \leq m)$. $U(\widetilde{u}, \lambda) \in A(2 m-1, m-1)$. Solving the adjoint representation equation (4), where

$$
V=\left(\begin{array}{ccc}
C & A+B & \rho \\
A-B & -C & \delta \\
\delta & -\rho & 0
\end{array}\right)
$$


with $A=\operatorname{diag}\left(A_{1}, \ldots, A_{m}\right), B=\operatorname{diag}\left(B_{1}, \ldots, B_{m}\right)$, $C=\operatorname{diag}\left(C_{1}, \ldots, C_{m}\right), \rho=\operatorname{diag}\left(\rho_{1}, \ldots, \rho_{m}\right)$, and $\delta=$ $\operatorname{diag}\left(\delta_{1}, \ldots, \delta_{m}\right)$, we have

$$
\begin{gathered}
A_{k, x}=2 r_{k} B_{k}-2 \lambda C_{k}-\alpha_{k} \rho_{k}+\beta_{k} \delta_{k}, \\
B_{k, x}=2 r_{k} A_{k}-2 s_{k} C_{k}-\alpha_{k} \rho_{k}-\beta_{k} \delta_{k}, \\
C_{k, x}=2 \lambda A_{k}-2 s_{k} B_{k}+\alpha_{k} \delta_{k}+\beta_{k} \rho_{k}, \\
\rho_{k, x}=\lambda \delta_{k}+r_{k} \rho_{k}+s_{k} \delta_{k} \\
\quad-C_{k} \alpha_{k}-\beta_{k} A_{k}-\beta_{k} B_{k}, \\
\delta_{k, x}=-\lambda \rho_{k}+s_{k} \rho_{k}-r_{k} \delta_{k} \\
-\alpha_{k} A_{k}+\alpha_{k} B_{k}+\beta_{k} C_{k},
\end{gathered}
$$

where $1 \leq k \leq m$. Let $A_{k}=\sum_{j \geq 0} A_{k}^{(j)} \lambda^{-j}, B_{k}=\sum_{j \geq 0} B_{k}^{(j)} \lambda^{-j}$, $C_{k}=\sum_{j \geq 0} C_{k}^{(j)} \lambda^{-j}, \rho_{k}=\sum_{j \geq 0} \rho_{k}^{(j)} \lambda^{-j}$, and $\delta_{k}=\sum_{j \geq 0} \delta_{k}^{(j)} \lambda^{-j}$, and then we have

$$
\begin{gathered}
A_{k}^{(0)}=C_{k}^{(0)}=\rho_{k}^{(0)}=\delta_{k}^{(0)}=0, \\
A_{k, x}^{(j)}=-2 C_{k}^{(j+1)}+2 r_{k} B_{k}^{(j)}-\alpha_{k} \rho_{k}^{(j)}+\beta_{k} \delta_{k}^{(j)}, \\
B_{k, x}^{(j)}=2 r_{k} A_{k}^{(j)}-2 s_{k} C_{k}^{(j)}-\alpha_{k} \rho_{k}^{(j)}-\beta_{k} \delta_{k}^{(j)}, \\
C_{k, x}^{(j)}=2 A_{k}^{(j+1)}-2 s_{k} B_{k}^{(j)}+\alpha_{k} \delta_{k}^{(j)}+\beta_{k} \rho_{k}^{(j)}, \\
\rho_{k, x}^{(j)}=\delta_{k}^{(j+1)}+r_{k} \rho_{k}^{(j)}+s_{k} \delta_{k}^{(j)} \\
-\alpha_{k} C_{k}^{(j)}-\beta_{k} A_{k}^{(j)}-\beta_{k} B_{k}^{(j)}, \\
\delta_{k, x}^{(j)}=-\rho_{k}^{(j+1)}+s_{k} \rho_{k}^{(j)}-r_{k} \delta_{k}^{(j)} \\
-\alpha_{k} A_{k}^{(j)}+\alpha_{k} B_{k}^{(j)}+\beta_{k} C_{k}^{(j)},
\end{gathered}
$$

where $j \geq 0,1 \leq k \leq m$, which can be written as a recursive form:

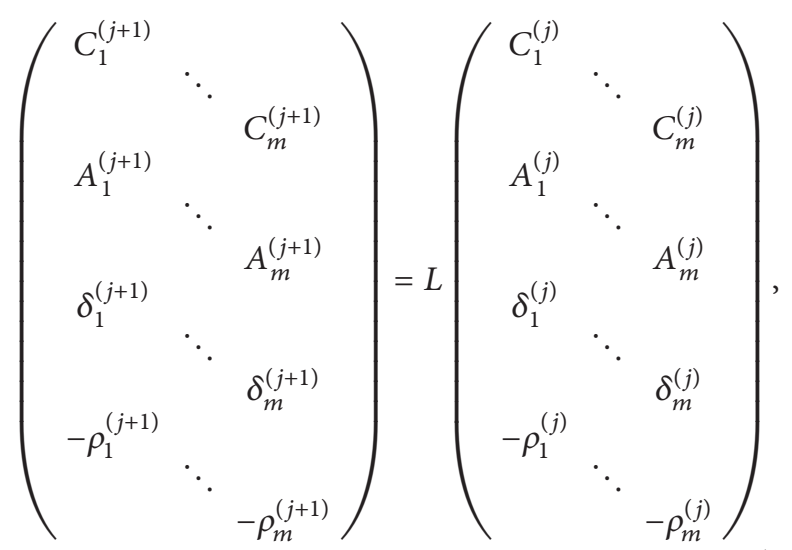

where the recursive operator is given by

$$
L=\left(\begin{array}{llll}
L_{11} & L_{12} & L_{13} & L_{14} \\
L_{21} & L_{22} & L_{23} & L_{24} \\
L_{31} & L_{32} & L_{33} & L_{34} \\
L_{41} & L_{42} & L_{43} & L_{44}
\end{array}\right),
$$

with

$$
\begin{gathered}
L_{11}=\operatorname{diag}\left(-2 r_{1} \partial^{-1} s_{1}, \ldots,-2 r_{m} \partial^{-1} s_{m}\right), \\
L_{12}=\operatorname{diag}\left(-\frac{1}{2} \partial+2 r_{1} \partial^{-1} r_{1}, \ldots,-\frac{1}{2} \partial+2 r_{m} \partial^{-1} r_{m}\right) \\
L_{13}=\operatorname{diag}\left(\frac{1}{2} \beta_{1}-r_{1} \partial^{-1} \beta_{1}, \ldots, \frac{1}{2} \beta_{m}-r_{m} \partial^{-1} \beta_{m}\right), \\
L_{14}=\operatorname{diag}\left(\frac{1}{2} \alpha_{1}+r_{1} \partial^{-1} \alpha_{1}, \ldots, \frac{1}{2} \alpha_{m}+r_{m} \partial^{-1} \alpha_{m}\right), \\
L_{21}=\operatorname{diag}\left(\frac{1}{2} \partial-2 s_{1} \partial^{-1} s_{1}, \ldots, \frac{1}{2} \partial-2 s_{m} \partial^{-1} s_{m}\right) \\
L_{22}=\operatorname{diag}\left(2 s_{1} \partial^{-1} r_{1}, \ldots, 2 s_{m} \partial^{-1} r_{m}\right) \\
L_{23}=\operatorname{diag}\left(-\frac{1}{2} \alpha_{1}-s_{1} \partial^{-1} \beta_{1}, \ldots,-\frac{1}{2} \alpha_{m}-s_{m} \partial^{-1} \beta_{m}\right), \\
L_{24}=\operatorname{diag}\left(\frac{1}{2} \beta_{1}+s_{1} \partial^{-1} \alpha_{1}, \ldots, \frac{1}{2} \beta_{m}+s_{m} \partial^{-1} \alpha_{m}\right), \\
L_{31}=\operatorname{diag}\left(\alpha_{1}-2 \beta_{1} \partial^{-1} s_{1}, \ldots, \alpha_{m}-2 \beta_{m} \partial^{-1} s_{m}\right) \\
L_{32}=\operatorname{diag}\left(\beta_{1}+2 \beta_{1} \partial^{-1} r_{1}, \ldots, \beta_{m}+2 \beta_{m} \partial^{-1} r_{m}\right), \\
L_{33}=\operatorname{diag}\left(-s_{1}-\beta_{1} \partial^{-1} \beta_{1}, \ldots,-s_{m}-\beta_{m} \partial^{-1} \beta_{m}\right), \\
L_{34}=\operatorname{diag}\left(-\partial+r_{1}+\beta_{1} \partial^{-1} \alpha_{1}, \ldots,-\partial+r_{m}+\beta_{m} \partial^{-1} \alpha_{m}\right), \\
L_{41}=\operatorname{diag}\left(-\beta_{1}+2 \alpha_{1} \partial^{-1} s_{1}, \ldots,-\beta_{m}+2 \alpha_{m} \partial^{-1} s_{m}\right), \\
L_{42}=\operatorname{diag}\left(\alpha_{1}-2 \alpha_{1} \partial^{-1} r_{1}, \ldots, \alpha_{m}-2 \alpha_{m} \partial^{-1} r_{m}\right) \\
L_{43}=\operatorname{diag}\left(\partial+r_{1}+\alpha_{1} \partial^{-1} \beta_{1}, \ldots, \partial+r_{m}+\alpha_{m} \partial^{-1} \beta_{m}\right), \\
L_{44}=\operatorname{diag}\left(s_{1}-\alpha_{1} \partial^{-1} \alpha_{1}, \ldots, s_{m}-\alpha_{m} \partial^{-1} \alpha_{m}\right)
\end{gathered}
$$

It is easy to verify that $B_{k, x}^{(0)}=0(1 \leq k \leq m)$. Choosing $B_{k}^{(0)}=1$ and constant of integration to be zero, the first few terms can be worked out as follows:

$$
\begin{gathered}
A_{k}^{(1)}=s_{k}, \quad B_{k}^{(1)}=0, \quad C_{k}^{(1)}=r_{k}, \\
\rho_{k}^{(1)}=\alpha_{k}, \quad \delta_{k}^{(1)}=\beta_{k}, \\
A_{k}^{(2)}=\frac{1}{2} r_{k, x}, \quad B_{k}^{(2)}=\frac{1}{2}\left(r_{k}^{2}+s_{k}^{2}\right)+\alpha_{k} \beta_{k}, \\
C_{k}^{(2)}=-\frac{1}{2} s_{k, x}, \quad \rho_{k}^{(2)}=-\beta_{k, x}, \quad \delta_{k}^{(2)}=\alpha_{k, x} .
\end{gathered}
$$

In what follows, the spectral problem (29) is associated with the auxiliary spectral problem:

$$
\phi_{t_{n}}=V^{(n)} \phi
$$

where 


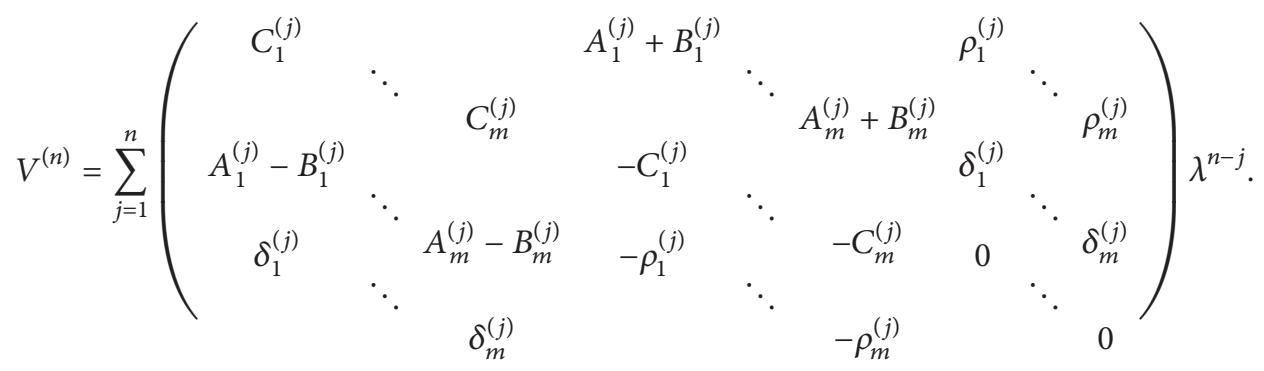

The compatibility condition between (29) and (37) gives the following super nonlinear soliton hierarchy:

$$
\begin{gathered}
r_{k, t_{n}}=2 A_{k}^{(n+1)}, \\
s_{k, t_{n}}=-2 C_{k}^{(n+1)}, \\
\alpha_{k, t_{n}}=\delta_{k}^{(n+1)}, \\
\beta_{k, t_{n}}=-\rho_{k}^{(n+1)},
\end{gathered}
$$

where $1 \leq k \leq m$. By denoting $A^{(j)}=\operatorname{diag}\left(A_{1}^{(j)}, \ldots, A_{m}^{(j)}\right)$, $B^{(j)}=\operatorname{diag}\left(B_{1}^{(j)}, \ldots, B_{m}^{(j)}\right), C^{(j)}=\operatorname{diag}\left(C_{1}^{(j)}, \ldots, C_{m}^{(j)}\right), \rho^{(j)}=$ $\operatorname{diag}\left(\rho_{1}^{(j)}, \ldots, \rho_{m}^{(j)}\right)$, and $\delta^{(j)}=\operatorname{diag}\left(\delta_{1}^{(j)}, \ldots, \delta_{m}^{(j)}\right)$, with $j \geq 0$, and (39) can be written as follows:

$$
\begin{gathered}
r_{t_{n}}=2 A^{(n+1),} \\
s_{t_{n}}=-2 C^{(n+1)}, \\
\alpha_{t_{n}}=\delta^{(n+1)}, \\
\beta_{t_{n}}=-\rho^{(n+1)} .
\end{gathered}
$$

Similarly, when $m=1,(40)$ is equivalent to the super Dirac soliton hierarchy $[4,30]$, and thus (40) is called multicomponent super Dirac hierarchy.

To derive super bi-Hamiltonian structure of (40), we need to use the supertrace identity (7). To this end, we firstly obtain the following equalities:

$$
\begin{gathered}
\operatorname{str}\left(V \frac{\partial U}{\partial \lambda}\right)=-2 \operatorname{tr} B, \quad \operatorname{str}\left(\frac{\partial U}{\partial s} V\right)=2 A, \\
\operatorname{str}\left(\frac{\partial U}{\partial r} V\right)=2 C, \quad \operatorname{str}\left(\frac{\partial U}{\partial \alpha} V\right)=2 \delta \\
\operatorname{str}\left(\frac{\partial U}{\partial \beta} V\right)=-2 \rho .
\end{gathered}
$$

Thus, the supertrace identity (7) becomes the following equality:

$$
\begin{aligned}
& \left(\begin{array}{ccc}
\frac{\delta}{\delta r_{1}} & & \\
& \ddots & \frac{\delta}{\delta r_{m}} \\
\frac{\delta}{\delta s_{1}} & & \\
& \ddots & \frac{\delta}{\delta s_{m}} \\
\frac{\delta}{\delta \alpha_{1}} & & \\
\frac{\delta}{\delta \beta_{1}} & & \\
& & \frac{\delta}{\delta \alpha_{m}} \\
& & \frac{\delta}{\delta \beta_{m}}
\end{array}\right) \int \sum_{k=1}^{m} B_{k}^{(j)} \lambda^{-j} d x \\
& =\left(\lambda^{-\gamma} \frac{\partial}{\partial \lambda} \lambda^{\gamma}\right)\left(\begin{array}{ccc}
\sum_{j \geq 0} C_{1}^{(j)} \lambda^{-j} & & \\
& \ddots & \\
\sum_{j \geq 0} A_{1}^{(j)} \lambda^{-j} & & \sum_{j \geq 0} C_{m}^{(j)} \lambda^{-j} \\
\sum_{j \geq 0} \delta_{1}^{(j)} \lambda^{-j} & & \sum_{j \geq 0} A_{m}^{(j)} \lambda^{-j} \\
& \ddots & \\
-\sum_{j \geq 0} \rho_{1}^{(j)} \lambda^{-j} & & \\
& \ddots & \\
& & -\sum_{j \geq 0} \rho_{m}^{(j)} \lambda^{-j}
\end{array}\right) .
\end{aligned}
$$

Equating the coefficients of $\lambda^{-j-2}$ in the above equality, we have 


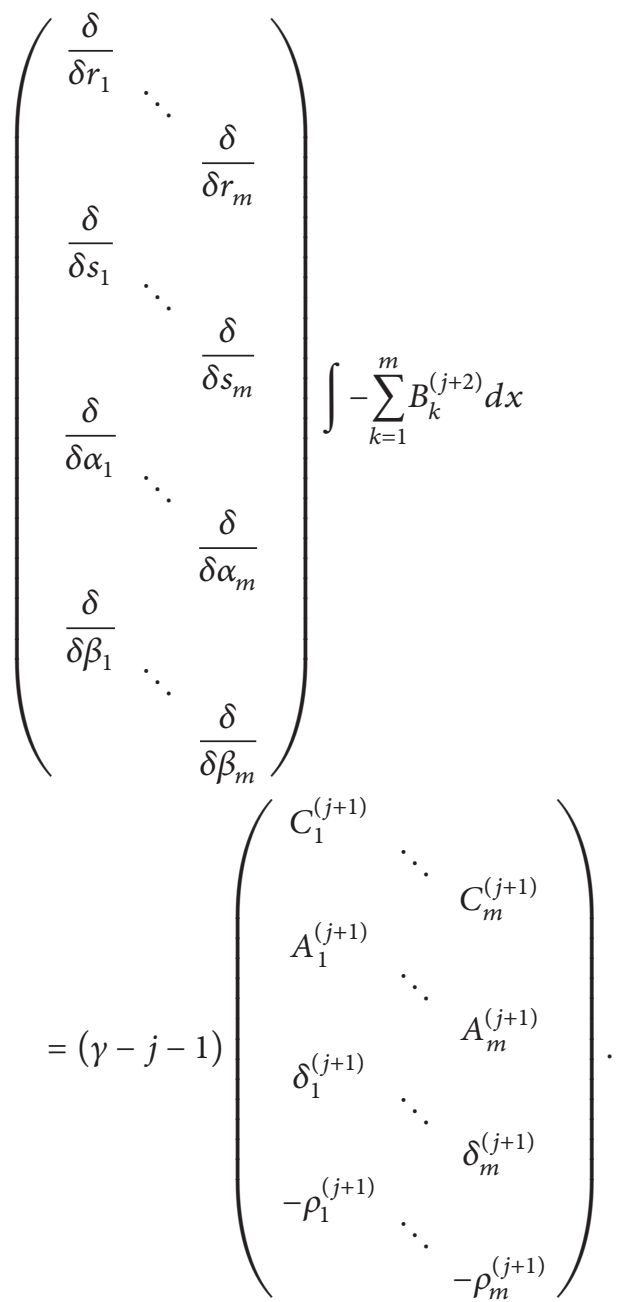

Let $j=0$, and we have $\gamma=0$. Thus, we obtain

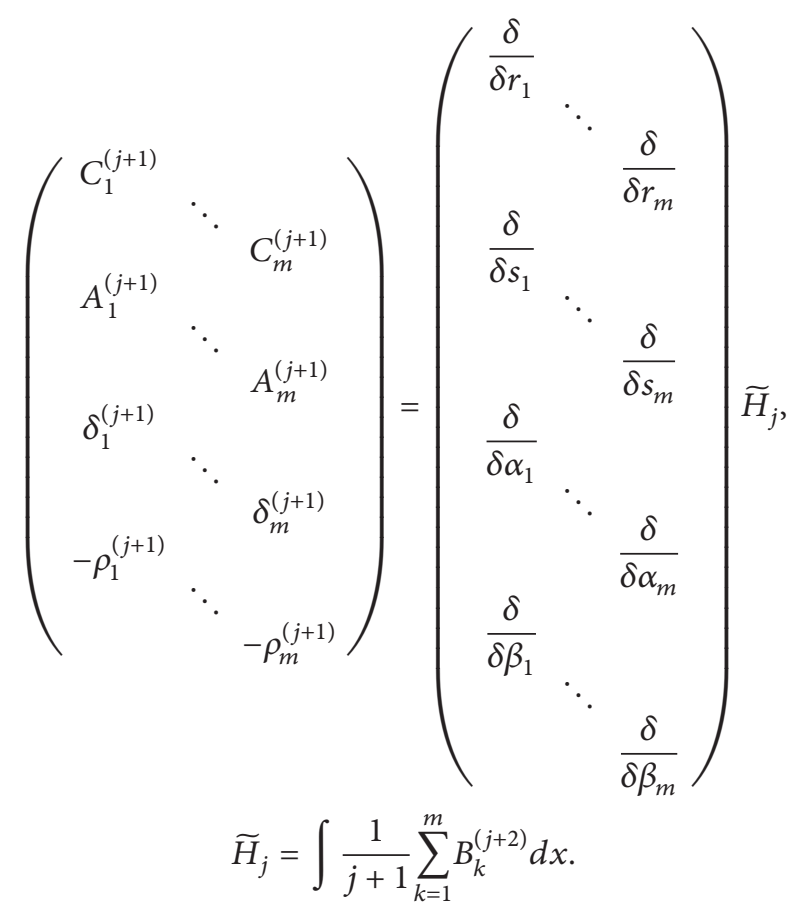

Therefore, (39) or (40) can be written in the following super bi-Hamiltonian structure:

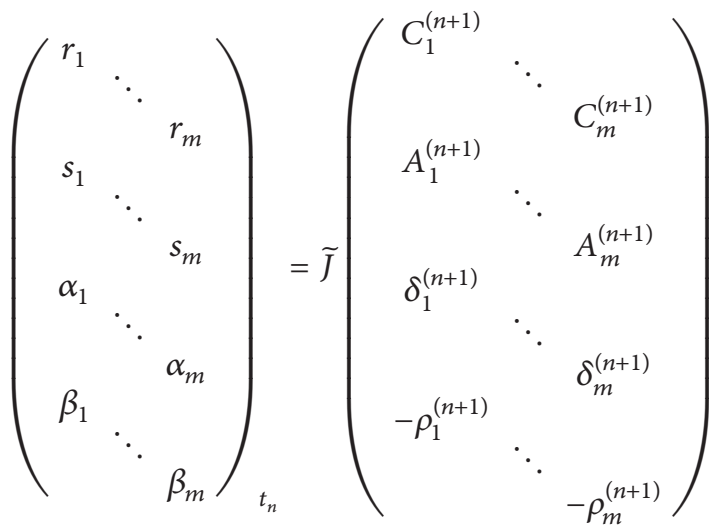

$$
\begin{aligned}
& =\widetilde{J} \frac{\delta}{\delta \widetilde{u}} \widetilde{H}_{n}=\widetilde{J} L \frac{\delta}{\delta \widetilde{u}} \widetilde{H}_{n-1},
\end{aligned}
$$

where $\widetilde{J}=\left(\begin{array}{cccc}0 & 2 E & 0 & 0 \\ -2 E & 0 & 0 & 0 \\ 0 & 0 & E & 0 \\ 0 & 0 & 0 & E\end{array}\right)$ is the supersymplectic operator.

Example 2. Let $m=2, n=2$ in (45), and we obtain twocomponent super Dirac equations:

$$
\begin{aligned}
r_{1, t_{2}}= & -\frac{1}{2} s_{1, x x}+r_{1}^{2} s_{1}+s_{1}^{3} \\
& +2 s_{1} \alpha_{1} \beta_{1}-\alpha_{1} \alpha_{1, x}+\beta_{1} \beta_{1, x}, \\
r_{2, t_{2}}= & -\frac{1}{2} s_{2, x x}+r_{2}^{2} s_{2}+s_{2}^{3} \\
& +2 s_{2} \alpha_{2} \beta_{2}-\alpha_{2} \alpha_{2, x}+\beta_{2} \beta_{2, x}, \\
s_{1, t_{2}}= & \frac{1}{2} r_{1, x x}-r_{1}^{3}-r_{1} s_{1}^{2}-2 r_{1} \alpha_{1} \beta_{1} \\
& -\alpha_{1} \beta_{1, x}+\alpha_{1, x} \beta_{1}, \\
s_{2, t_{2}}= & \frac{1}{2} r_{2, x x}-r_{2}^{3}-r_{2} s_{2}^{2}-2 r_{2} \alpha_{2} \beta_{2} \\
& -\alpha_{2} \beta_{2, x}+\alpha_{2, x} \beta_{2}, \\
\alpha_{1, t_{2}}= & -\beta_{1, x x}+r_{1} \beta_{1, x}-s_{1} \alpha_{1, x}-\frac{1}{2} \alpha_{1} s_{1, x} \\
\beta_{1, t_{2}}= & \alpha_{1, x x}+s_{1} \beta_{1, x}+r_{1} \alpha_{1, x}+\frac{1}{2} \alpha_{1} r_{1, x} \\
& +\frac{1}{2} \beta_{1} r_{1, x}+\frac{1}{2} r_{1}^{2} \beta_{1}+\frac{1}{2} s_{1}^{2} \beta_{1}, \\
\alpha_{2, t_{2}}= & -\beta_{2, x x}+r_{2} \beta_{2, x}-s_{2} \alpha_{2, x}-\frac{1}{2} \alpha_{2} s_{2, x} \\
& +\frac{1}{2} \beta_{2} r_{2, x}+\frac{1}{2} r_{2}^{2} \beta_{2}+\frac{1}{2} s_{2}^{2} \beta_{2}, \\
2 & \frac{1}{2} \beta_{1} s_{1, x}, \\
& \\
& \\
&
\end{aligned}
$$




$$
\begin{aligned}
\beta_{2, t_{2}}= & \alpha_{2, x x}+s_{2} \beta_{2, x}+r_{2} \alpha_{2, x}+\frac{1}{2} \alpha_{2} r_{2, x} \\
& -\frac{1}{2} r_{2}^{2} \alpha_{2}-\frac{1}{2} s_{2}^{2} \alpha_{2}+\frac{1}{2} \beta_{2} s_{2, x} .
\end{aligned}
$$

\section{Conclusions and Discussions}

Starting from the matrix Lie superalgebra $A(2 m-1, m-1)$, we have, respectively, constructed multicomponent super AKNS equations (21) and multicomponent super Dirac equations (40). By making use of the supertrace identity (7), (21), and (40) have been rewritten as integrable super-Hamiltonian forms (26) and (45), respectively. Moreover, we believe that many multicomponent superintegrable equations can also be constructed, which may be helpful to many physical and mathematical researchers in their future work.

\section{Acknowledgments}

This work is supported by the National Natural Science Foundation of China under Grant nos. 10971109, 11001069, 11271210, and 61273077 and Zhejiang Provincial Natural Science Foundation of China under Grant nos. LQ12A01002 and LQ12A01003.

\section{References}

[1] G. Z. Tu, "The trace identity, a powerful tool for constructing the Hamiltonian structure of integrable systems," Journal of Mathematical Physics, vol. 30, no. 2, pp. 330-338, 1989.

[2] X.-B. Hu, "An approach to generate superextensions of integrable systems," Journal of Physics A, vol. 30, no. 2, pp. 619-632, 1997.

[3] X. B. Hu, Integrable systems and related problems [Doctoral dissertation], Computing Center of Academia Sinica, 1990.

[4] W.-X. Ma, J.-S. He, and Z.-Y. Qin, "A supertrace identity and its applications to superintegrable systems," Journal of Mathematical Physics, vol. 49, no. 3, Article ID 033511, 13 pages, 2008.

[5] J. Yu, J. He, Y. Cheng, and J. Han, "A novel symmetry constraint of the super cKdV system," Journal of Physics A, vol. 43, no. 44, Article ID 445201, 12 pages, 2010.

[6] S.-X. Tao and T.-C. Xia, "Lie algebra and lie super algebra for integrable couplings of C-KdV hierarchy," Chinese Physics Letters, vol. 27, no. 4, Article ID 040202, 4 pages, 2010.

[7] X.-G. Geng and L.-H. Wu, "A super extension of Kaup-Newell hierarchy," Communications in Theoretical Physics, vol. 54, no. 4, pp. 594-598, 2010.

[8] S.-X. Tao, T.-C. Xia, and H. Shi, "Super-KN hierarchy and its super-Hamiltonian structure," Communications in Theoretical Physics, vol. 55, no. 3, pp. 391-395, 2011.

[9] M. Vijayajayanthi, T. Kanna, and M. Lakshmanan, "Bright-dark solitons and their collisions in mixed $\mathrm{N}$-coupled nonlinear Schrödinger equations," Physical Review A, vol. 77, no. 1, Article ID 013820, 18 pages, 2008.

[10] S. Rajendran, P. Muruganandam, and M. Lakshmanan, "Interaction of dark-bright solitons in two-component Bose-Einstein condensates," Journal of Physics B, vol. 42, no. 14, Article ID 145307, 5 pages, 2009.

[11] Q.-H. Park and H. J. Shin, "Painlevé analysis of the coupled nonlinear Schrödinger equation for polarized optical waves in an isotropic medium," Physical Review E, vol. 59, no. 2, pp. 23732379, 1999.

[12] W. Ma and R. Zhou, "Adjoint symmetry constraints of multicomponent AKNS equations," Chinese Annals of Mathematics B, vol. 23, no. 3, pp. 373-384, 2002.

[13] Y. Zhang, "A multi-component matrix loop algebra and a unified expression of the multi-component AKNS hierarchy and the multi-component BPT hierarchy," Physics Letters A, vol. 342, no. 1-2, pp. 82-89, 2005.

[14] A. P. Fordy and P. P. Kulish, "Nonlinear Schrödinger equations and simple Lie algebras," Communications in Mathematical Physics, vol. 89, no. 3, pp. 427-443, 1983.

[15] T. Tsuchida, H. Ujino, and M. Wadati, "Integrable semidiscretization of the coupled modified KdV equations," Journal of Mathematical Physics, vol. 39, no. 9, pp. 4785-4813, 1998.

[16] T. Tsuchida and M. Wadati, "New integrable systems of derivative nonlinear Schrödinger equations with multiple components," Physics Letters A, vol. 257, no. 1-2, pp. 53-64, 1999.

[17] W.-X. Ma and R. Zhou, "Adjoint symmetry constraints leading to binary nonlinearization," Journal of Nonlinear Mathematical Physics, vol. 9, supplement 1, pp. 106-126, 2002.

[18] W.-X. Ma, "Multi-component bi-Hamiltonian Dirac integrable equations," Chaos, Solitons \& Fractals, vol. 39, no. 1, pp. 282-287, 2009.

[19] V. S. Gerdzhikov, G. G. Grakhovski, and N. A. Kostov, "Multicomponent equations of the nonlinear Schrödinger type on symmetric spaces and their reductions," Theoretical and Mathematical Physics, vol. 144, no. 2, pp. 1147-1156, 2005.

[20] V. S. Gerdjikov and N. A. Kostov, "Reductions of multicomponent $\mathrm{mKdV}$ equations on symmetric spaces of DIII-type," Symmetry, Integrability and Geometry: Methods and Applications, vol. 4, article 029, 30 pages, 2008.

[21] Y.-P. Sun and H.-W. Tam, "A hierarchy of non-isospectral multicomponent AKNS equations and its integrable couplings," Physics Letters A, vol. 370, no. 2, pp. 139-144, 2007.

[22] H. Dong and X. Gong, "A (2+1)-dimensional multi-component AKNS integrable hierarchy and its expanding model," Chaos, Solitons \& Fractals, vol. 33, no. 3, pp. 945-950, 2007.

[23] F. Yu and L. Li, "A new matrix Lie algebra, the multicomponent Yang hierarchy and its super-integrable coupling system," Applied Mathematics and Computation, vol. 207, no. 2, pp. 380387, 2009.

[24] L. A. Dickey, "Additional symmetries of KP, Grassmannian, and the string equation. II," Modern Physics Letters A, vol. 8, no. 14, pp. 1357-1377, 1993.

[25] H.-X. Wu, X.-J. Liu, and Y.-B. Zeng, "Two new multicomponent BKP hierarchies," Communications in Theoretical Physics, vol. 51, no. 2, pp. 193-199, 2009.

[26] H. Z. Sun and Q. Z. Han, Lie Algebras and Lie Superalgebras and Their Applications in Physics, Peking University Press, Beijing, China, 1999.

[27] Y. S. Li and L. N. Zhang, "Super AKNS scheme andits infinite conserved currents," Il Nuovo Cimento A, vol. 93, no. 2, pp. 175183, 1986.

[28] Y. S. Li and L. N. Zhang, "A note on the super AKNS equations," Journal of Physics A, vol. 21, no. 7, pp. 1549-1552, 1988. 
[29] Y. S. Li and L. N. Zhang, "Hamiltonian structure of the super evolution equation," Journal of Mathematical Physics, vol. 31, no. 2, pp. 470-475, 1990.

[30] J. He, J. Yu, Y. Cheng, and R. Zhou, "Binary nonlinearization of the super AKNS system," Modern Physics Letters B, vol. 22, no. 4, pp. 275-288, 2008. 


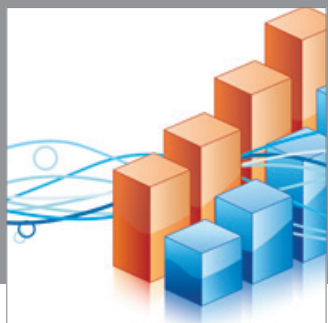

Advances in

Operations Research

mansans

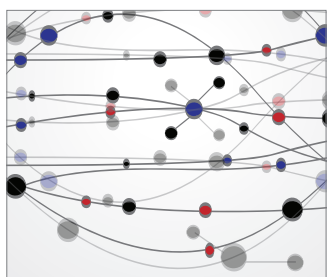

The Scientific World Journal
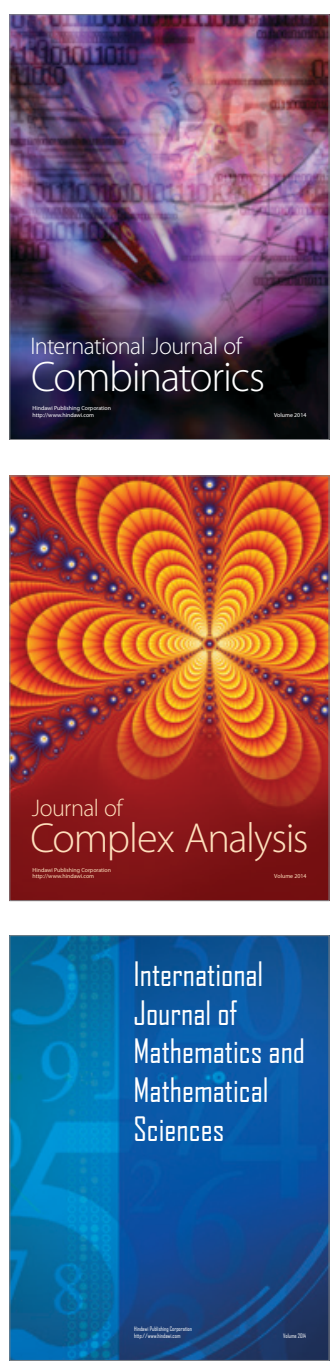
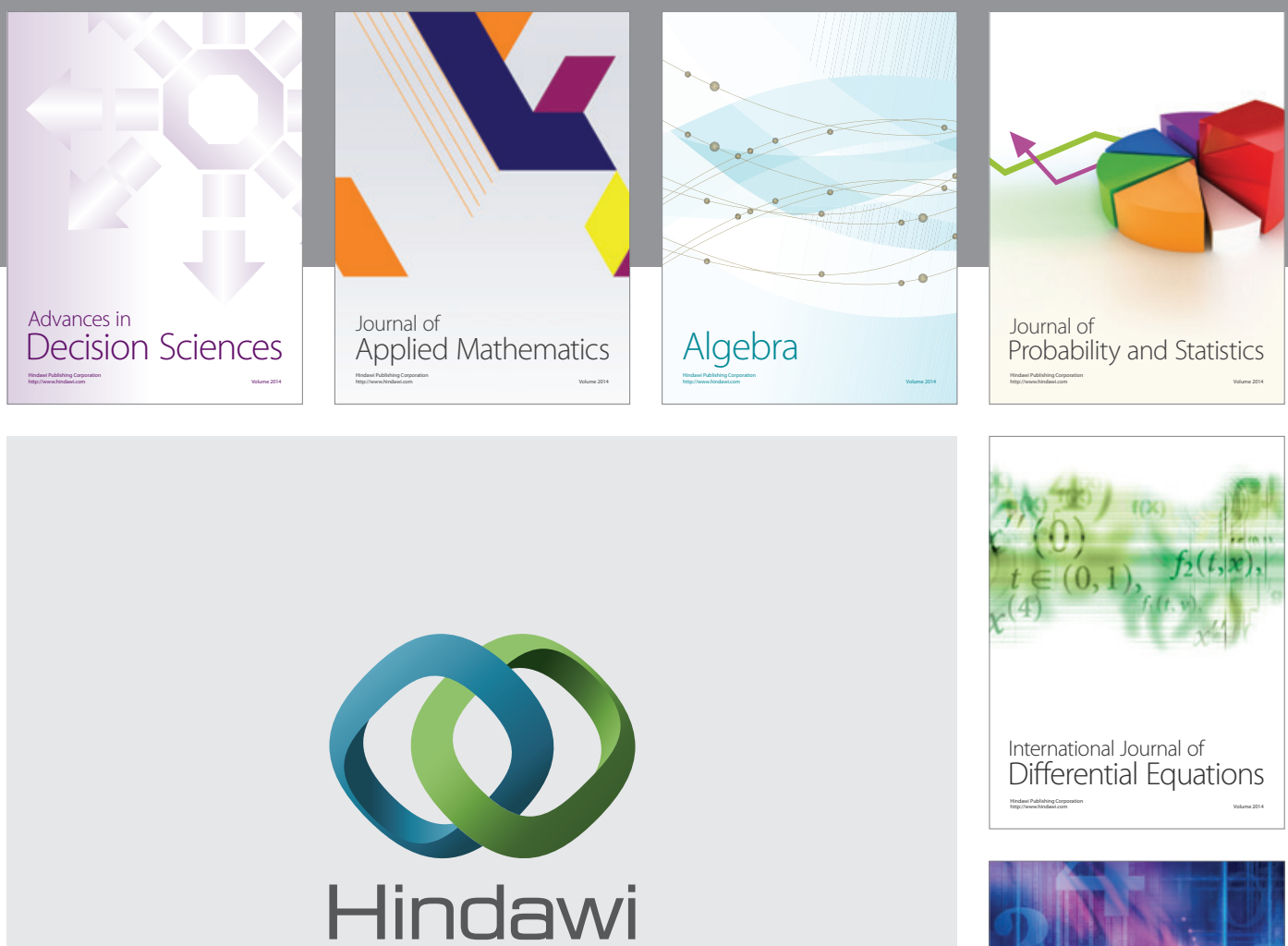

Submit your manuscripts at http://www.hindawi.com
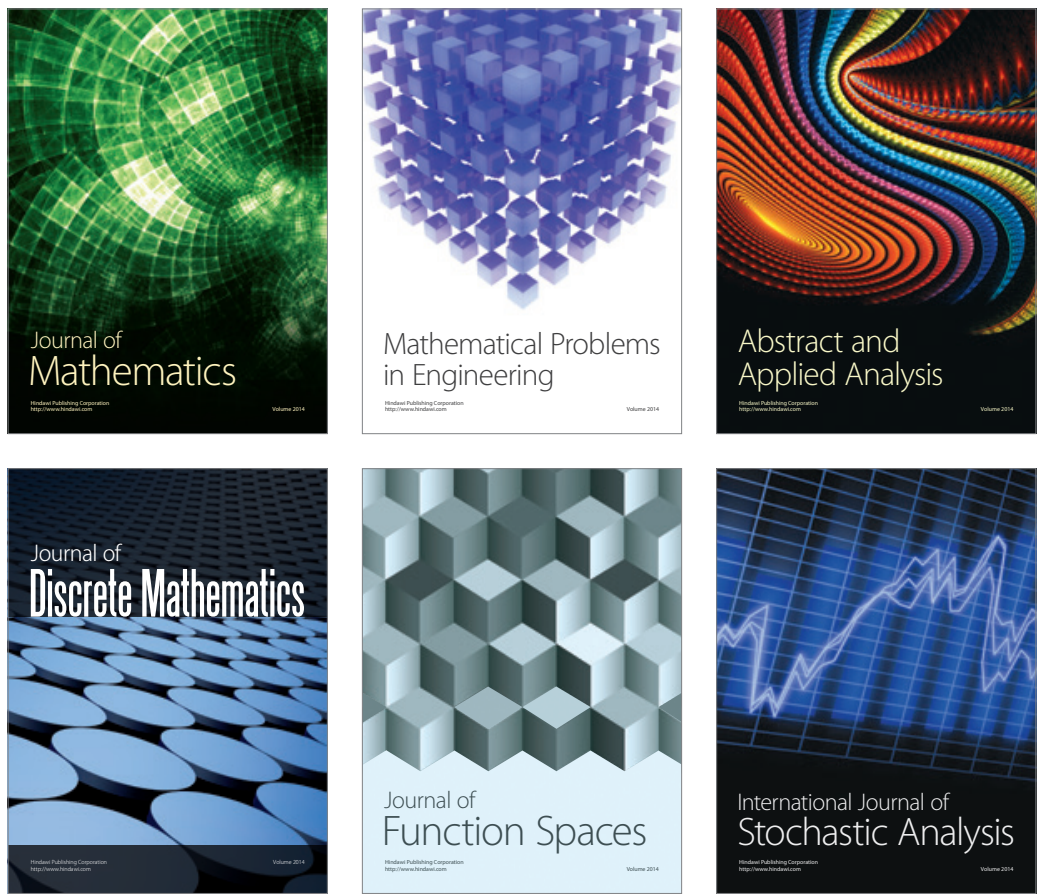

Journal of

Function Spaces

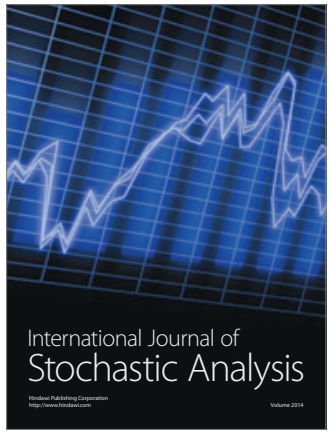

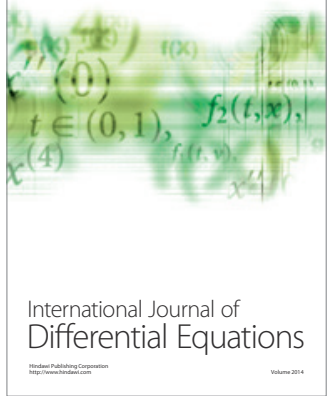
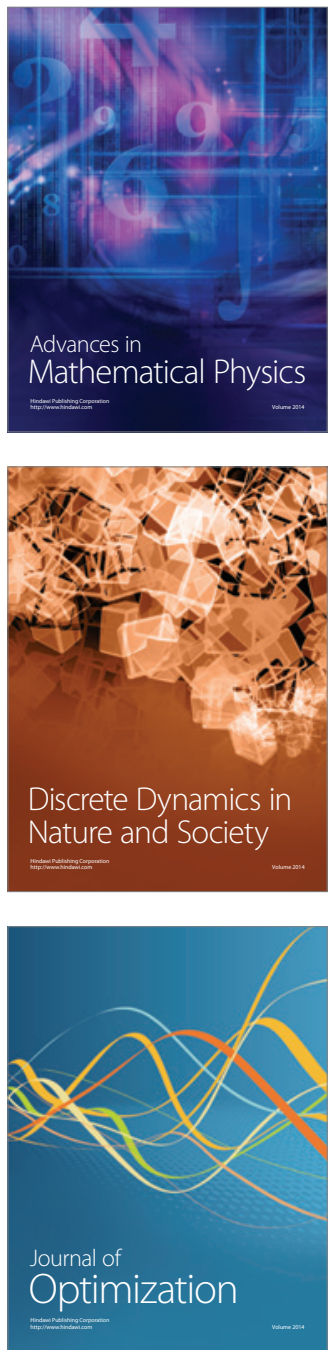